\title{
THE PHASE REPRESENTATION OF COVARIANT PHASE OBSERVABLES
}

\author{
JUHA-PEKKA PELLONPÄÄ
}

\begin{abstract}
Covariant phase observables are obtained by defining simple conditions for mappings from the set of phase wave functions (unit vectors of the Hardy space) to the set of phase probability densities. The existence of phase probability density for any phase wave function, the existence of interference effects, and the natural phase shift covariance are those simple conditions. The nonlocalizability of covariant phase observables is proved.
\end{abstract}

PACS numbers: 42.50.Dv, 03.65.w

Keywords: phase observable, phase shift covariance, phase measurement, Hardy space

Let us start with some necessary conditions of phase measurements. From a phase measurement one gets phase statistics, that is, a probability density $f$ defined on the phase interval, say, $[0,2 \pi)$. Thus, $(2 \pi)^{-1} \int_{0}^{2 \pi} f(\theta) \mathrm{d} \theta=1, f(\theta) \geq 0, \theta \in[0,2 \pi)$, and $f$ can be expanded periodically to the whole real line. The probability for phase to be in the phase window $X \subseteq[0,2 \pi)$ is $(2 \pi)^{-1} \int_{X} f(\theta) \mathrm{d} \theta$.

In quantum theory, one associates a Hilbert space to the physical system under consideration. In the case of a single mode optical field, it is convenient to choose the number space $\mathcal{H}_{N}$ spanned by number states $(|n\rangle)_{n \in \mathbb{N}}$ to describe the system. The number space is isomorphic to the Hardy class $H^{2}$ on the unit disc via the mapping $|n\rangle \mapsto e^{-i n \theta}$. Hence, an element $\psi$ of $H^{2}$ can be represented as a Fourier series $\psi(\theta)=\sum_{n=0}^{\infty} d_{n} e^{-i n \theta}$ where $\sum_{n=0}^{\infty}\left|d_{n}\right|^{2}<\infty$. The Hardy class $H^{2}$ is a subspace of $L^{2}([0,2 \pi))$, the $L^{2}([0,2 \pi))$-space consisting of all square integrable functions $[0,2 \pi) \rightarrow \mathbb{C}$ with Fourier-series $\sum_{n=-\infty}^{\infty} d_{n} e^{-i n \theta}$ where $\sum_{n=-\infty}^{\infty}\left|d_{n}\right|^{2}<\infty$. 
The number operator $N=\sum_{n=0}^{\infty} n|n\rangle\langle n|$ is isomorphic to the derivative operator $i \partial_{\theta}$ in $H^{2}$, and the phase shifter $e^{i \alpha N}$ operates as $\psi(\theta) \mapsto \psi(\theta-\alpha)$ in $H^{2}$. It is natural to say that $H^{2}$ is a phase representation space for the single mode system, and we call (normalized) elements of $\mathrm{H}^{2}$ as phase wave functions.

Suppose that the single mode system is prepared in a (vector) state $\psi \in H^{2},\|\psi\|=1$, and we make phase measurements and get a phase probability density $f$. The canonical distribution is $f(\theta)=|\psi(\theta)|^{2}$ and the trivial (random) distribution is $f(\theta)=1$. Next we state general conditions for phase measurements.

1. Existence of probability densities. For any phase wave function $\psi \in H^{2}$ there exists a phase probability density $f_{\psi, \psi}$ : $[0,2 \pi) \rightarrow[0, \infty]$

2. Existence of interference effects. For any two phase wave functions $\psi, \varphi \in H^{2}$ there exist complex valued functions $f_{\psi, \varphi}$ and $f_{\varphi, \psi}$ defined on $[0,2 \pi)$ such that

$f_{c_{1} \psi+c_{2} \varphi, c_{1} \psi+c_{2} \varphi}(\theta)=\left|c_{1}\right|^{2} f_{\psi, \psi}(\theta)+\left|c_{2}\right|^{2} f_{\varphi, \varphi}(\theta)+\overline{c_{1}} c_{2} f_{\psi, \varphi}(\theta)+c_{1} \overline{c_{2}} f_{\varphi, \psi}(\theta)$ for all $c_{1}, c_{2} \in \mathbb{C}$ such that $\left\|c_{1} \psi+c_{2} \varphi\right\|=1$.

3. Phase shift covariance. If one shifts the phase of a phase wave function $\psi$, that is, $\psi(\theta) \mapsto \psi^{\alpha}(\theta):=\psi(\theta+\alpha)$, then the probability density $f_{\psi, \psi}$ should only move without deforming its shape. Hence,

$$
f_{\psi^{\alpha}, \psi^{\alpha}}(\theta)=f_{\psi, \psi}(\theta+\alpha)
$$

for all $\theta, \alpha \in \mathbb{R}$.

Anticipating the below results, we say that the mapping $(\psi, \varphi) \mapsto f_{\psi, \varphi}$, which satisfies Conditions 1,2 , and 3 , is a phase observable. It follows from Condition 2 that any phase observable has a unique sesquilinear extension to $H^{2} \times H^{2}$. From Condition 1 one sees that this mapping is bounded (in the $L^{1}$-norm). Thus, from Proposition 2 of [1] one gets

$$
f_{\psi, \varphi}=\sum_{n, m=0}^{\infty} f_{\eta_{n}, \eta_{m}} \overline{a_{n}} b_{m}
$$

where $\eta_{n}(\theta):=e^{-i n \theta}, \psi(\theta)=\sum_{n=0}^{\infty} a_{n} e^{-i n \theta}$, and $\varphi(\theta)=\sum_{n=0}^{\infty} b_{n} e^{-i n \theta}$. Since

$$
\eta_{n}^{\alpha}(\theta):=\eta_{n}(\theta+\alpha)=e^{-i n \alpha} \eta_{n}(\theta)
$$

and due to the sesquilinearity

$$
f_{\eta_{n}^{\alpha}, \eta_{m}^{\alpha}}=e^{i(n-m) \alpha} f_{\eta_{n}, \eta_{m}}
$$

it follows from Condition 3 that

$$
f_{\eta_{n}, \eta_{m}}(\alpha)=f_{\eta_{n}^{\alpha}, \eta_{m}^{\alpha}}(0)=e^{i(n-m) \alpha} c_{n, m}
$$


where $c_{n, m}:=f_{\eta_{n}, \eta_{m}}(0)$. In order that $\theta \mapsto f_{\varphi, \varphi}(\theta)$ is positive and normalized, it is necessary that $\left(c_{n, m}\right)$ is positive semidefinite matrix with diagonals equal one, that is, $\left(c_{n, m}\right)$ is a phase matrix [2]. Hence, we can write (see Section 8 of [1]) that

$$
f_{\psi, \varphi}(\theta)=\sum_{n, m=0}^{\infty} c_{n, m} e^{i(n-m) \theta} \overline{a_{n}} b_{m}
$$

and

$$
\frac{1}{2 \pi} \int_{X} f_{\psi, \varphi}(\theta) \mathrm{d} \theta=\sum_{n, m=0}^{\infty} c_{n, m} \frac{1}{2 \pi} \int_{X} e^{i(n-m) \theta} \mathrm{d} \theta \overline{a_{n}} b_{m}
$$

for all (Borel) sets $X \subseteq[0,2 \pi)$.

For the canonical distribution, $f_{\psi, \varphi}(\theta)=\overline{\psi(\theta)} \varphi(\theta), c_{n, m}=1$ for all $n, m$, and for the trivial distribution $f_{\psi, \varphi}(\theta)=(2 \pi)^{-1} \int_{0}^{2 \pi} \overline{\psi(\theta)} \varphi(\theta) \mathrm{d} \theta$, $c_{n, m}=\delta_{n, m}$ (Kronecker's delta). Note that the mapping $(\psi, \varphi) \mapsto f_{\psi, \varphi}$ can be extended to the linear mapping from the set of trace-class operators on $H^{2}$ to the set of integrable functions $L^{1}([0,2 \pi)$ ) (see Proposition 2 of [1]). Especially, for any state (i.e., a positive trace-one operator) one gets a probability density function and, thus, a probability measure. A mapping from the set of states to the set of probability measures (on the outcome space of a measurement) defines a unique normalized positive operator measure $(\mathrm{POM})$ [3] and, thus, it is reasonable to call the mapping $(\psi, \varphi) \mapsto f_{\psi, \varphi}$ an observable.

A phase matrix $\left(c_{n, m}\right)$ defines a unique POM, a (covariant) phase observable [4, 5]

$$
E(X)=\sum_{n, m=0}^{\infty} c_{n, m} \frac{1}{2 \pi} \int_{X} e^{i(n-m) \theta} \mathrm{d} \theta|n\rangle\langle m|
$$

for all (Borel) sets $X \subseteq[0,2 \pi)$. Thus, using a unitary mapping $U$ : $\mathcal{H}_{N} \rightarrow H^{2},|n\rangle \mapsto U(|n\rangle):=\eta_{n}$,

$$
\frac{1}{2 \pi} \int_{X} f_{\psi, \varphi}(\theta) \mathrm{d} \theta=\left\langle\psi \mid U E(X) U^{*} \varphi\right\rangle, \quad \psi, \varphi \in H^{2},
$$

which shows that we can understand a phase observable as a phase shift covariant POM $E$, or a mapping $(\psi, \varphi) \mapsto f_{\psi, \varphi}$ satisfying conditions 1 , 2 , and 3 .

Theorem 2 of $[1]$ states that

$$
\left.E(X)=\sum_{n=0}^{\infty} V_{n} \frac{1}{2 \pi} \int_{X} \mid \theta\right)\left(\theta \mid \mathrm{d} \theta V_{n}^{*}\right.
$$

where $\mid \theta):=\sum_{n=0}^{\infty} e^{i n \theta}|n\rangle$ is the London phase state, and $V_{n}=$ $\sum_{k=0}^{\infty} z_{n, k}|k\rangle\langle k|$ are bounded operators for which $\sum_{n=0}^{\infty}\left|z_{n, k}\right|^{2}=1$ for 
all $k \in \mathbb{N}$. The mapping $\left.X \mapsto(2 \pi)^{-1} \int_{X} \mid \theta\right)(\theta \mid \mathrm{d} \theta$ is the canonical phase observable [4, 2]. Note that $\left\|V_{n}\right\| \leq 1$ and thus $\left\|V_{n} \psi\right\| \leq\|\psi\|$. In the case of the canonical phase, $V_{0}=I$ and $V_{n}=O, n \geq 1$, whereas in the case of the trivial phase, $V_{n}=|n\rangle\langle n|$ for all $n$. The canonical phase is (up to unitary equivalence) the only phase observable which is detemined by only one phase state $\left.V_{n} \mid \theta\right)$. Defining contractions $v_{n}:=U V_{n}^{*} U^{*}$ we may write

$$
\frac{1}{2 \pi} \int_{X} f_{\psi, \varphi}(\theta) \mathrm{d} \theta=\sum_{n=0}^{\infty} \frac{1}{2 \pi} \int_{X} \overline{v_{n} \psi(\theta)} v_{n} \varphi(\theta) \mathrm{d} \theta, \quad \psi, \varphi \in H^{2}
$$

For any $s \in \mathbb{N}$ we may define a continuous function $(x, y) \mapsto$ $C_{s}(x, y):=\sum_{n, m=0}^{s} e^{-i n x} c_{n, m} e^{i m y}$. Thus, one gets

$\frac{1}{2 \pi} \int_{X} f_{\varphi, \psi}(\theta) \mathrm{d} \theta=\lim _{s \rightarrow \infty} \frac{1}{2 \pi} \int_{X}\left[\frac{1}{2 \pi} \int_{0}^{2 \pi} \frac{1}{2 \pi} \int_{0}^{2 \pi} \overline{\varphi(x)} C_{s}(x-\theta, y-\theta) \psi(y) \mathrm{d} x \mathrm{~d} y\right] \mathrm{d} \theta$.

In the case of the canonical phase we may formally write that

$$
\lim _{s \rightarrow \infty} C_{s}(x, y) \rightarrow 2 \pi \delta_{2 \pi}(x) 2 \pi \delta_{2 \pi}(y)
$$

where $\delta_{2 \pi}$ is the $2 \pi$-periodic Dirac delta function!. In the case of the trivial phase

$$
\lim _{s \rightarrow \infty} C_{s}(x, y) \rightarrow 2 \pi \delta_{2 \pi}(x-y) .
$$

Hence, the canonical phase has the sharpest kernel $\lim _{s \rightarrow \infty} C_{s}$ and the trivial phase kernel loses all phase information of states. Note also that $C_{s}(0,0) \leq(s+1)^{2}$ and only in the case of the canonical phase $C_{s}(0,0)=(s+1)^{2}$ for all $s$.

Theorem 1. Let $(\psi, \varphi) \mapsto f_{\psi, \varphi}$ be a phase observable. For any (Borel) $X \subseteq[0,2 \pi)$ for which $\int_{X} \mathrm{~d} \theta<2 \pi$,

$$
\frac{1}{2 \pi} \int_{X} f_{\psi, \psi}(\theta) \mathrm{d} \theta<1
$$

for all unit vectors $\psi \in H^{2}$.

Proof. Let $\psi \in H^{2}$ be a unit vector. It follows from Theorem 3 of [1] that $(2 \pi)^{-1} \int_{X} f_{\psi, \psi}(\theta) \mathrm{d} \theta=\left\langle U^{*} \psi \mid E(X) U^{*} \psi\right\rangle=$ $(2 \pi)^{-1} \int_{X}\left(\theta\left|\Phi\left(\left|U^{*} \psi\right\rangle\left\langle U^{*} \psi\right|\right)\right| \theta\right) \mathrm{d} \theta$ where $\Phi$ is a covariant tracepreserving operation. Since $\Phi\left(\left|U^{*} \psi\right\rangle\left\langle U^{*} \psi\right|\right)=\sum_{k=0}^{\infty} \lambda_{k}\left|\varphi_{k}\right\rangle\left\langle\varphi_{k}\right|$, $\lambda_{k} \in[0,1], \varphi_{k} \in \mathcal{H}_{N},\left\|\varphi_{k}\right\|=1, k \in \mathbb{N}, \sum_{k=0}^{\infty} \lambda_{k}=1$, we get

${ }^{1}$ Note that formally $\delta_{2 \pi}(x)=(2 \pi)^{-1} \sum_{n=-\infty}^{\infty} e^{i n x}$ operating in a suitable subset of $L^{2}([0,2 \pi))$ as an integral $\left(f \mapsto \lim _{s \rightarrow \infty} \int_{0}^{2 \pi} f(x)(2 \pi)^{-1} \sum_{n=-s}^{s} e^{i n x} \mathrm{~d} x=f(0)\right)$. If we restrict the mapping $f \mapsto f(0)$ to the subset of $H^{2}$ then formally writing $\delta_{2 \pi}(x)=(2 \pi)^{-1} \sum_{n=0}^{\infty} e^{i n x}$. 
THE PHASE REPRESENTATION OF COVARIANT PHASE OBSERVABLES

$(2 \pi)^{-1} \int_{X} f_{\psi, \psi}(\theta) \mathrm{d} \theta=\sum_{k=0}^{\infty} \lambda_{k}(2 \pi)^{-1} \int_{X}\left|\left(\left.\theta\left|\varphi_{k}\right\rangle\right|^{2} \mathrm{~d} \theta\right.\right.$. From Proposition 8 of [6] it follows that $(2 \pi)^{-1} \int_{X}\left|\left(\left.\theta\left|\varphi_{k}\right\rangle\right|^{2} \mathrm{~d} \theta<1\right.\right.$ for any (Borel) $X \subseteq[0,2 \pi)$ for which $\int_{X} \mathrm{~d} \theta<2 \pi$ and this completes the proof.

Theorem 11 shows that we cannot find a phase wave function which is localized in some phase window $X$ (essentially) other than $[0,2 \pi)$, that is, a phase observable cannot be localized. Also this shows that any phase observable $E$ and the number $N$ are probabilistically complementary [6].

Remark 1. The first moment operator

$$
\left.A:=\frac{1}{2 \pi} \int_{0}^{2 \pi} \theta \mid \theta\right)\left(\theta\left|\mathrm{d} \theta=\pi I+\sum_{n \neq m=0}^{\infty} \frac{i}{m-n}\right| n\right\rangle\langle m|
$$

of the canonical phase observable

$$
\left.X \mapsto \frac{1}{2 \pi} \int_{X} \mid \theta\right)\left(\theta\left|\mathrm{d} \theta=\sum_{n, m=0}^{\infty} \frac{1}{2 \pi} \int_{X} e^{i(n-m) \theta} \mathrm{d} \theta\right| n\right\rangle\langle m|
$$

acts on the Hardy class $H^{2}$ as

$$
\psi \mapsto P Q \psi
$$

where $(Q \psi)(\theta)=\theta \psi(\theta)$ and $P$ is projection from $L^{2}([0,2 \pi))$ to $H^{2}$. Thus, $A$ is the Toeplitz phase operator suggested by Garrison and Wong [7] and also Galindo [8] and Mlak [9]. The canonical phase is (essentially) the only phase observable which has a covariant projection valued dilatation to $L^{2}([0,2 \pi))$ 10. The dilatation is the spectral measure $X \mapsto \chi_{X}$ of the multiplication operator $Q$.

Acknowledgments. The author thanks Dr. Pekka J. Lahti for many discussions, and for the careful reading of the article.

\section{REFERENCES}

[1] J.-P. Pellonpää, "On the structure of covariant phase observables," quant-ph/0108105, submitted to J. Math. Phys.

[2] P. J. Lahti and J.-P. Pellonpää, "Characterizations of the canonical phase observable," J. Math. Phys. 41, 7352-7381 (2000).

[3] P. Busch, M. Grabowski, and P. J. Lahti, Operational Quantum Physics (Springer-Verlag, Berlin, 1995).

[4] A. S. Holevo, Probabilistic and Statistical Aspects of Quantum Theory (North-Holland, Amsterdam, 1982).

[5] P. J. Lahti and J.-P. Pellonpää, "Covariant phase observables in quantum mechanics," J. Math. Phys. 40, 4688-4698 (1999).

[6] P. Busch, P. J. Lahti, J.-P. Pellonpää, and K. Ylinen, "Are number and phase complementary observables?" J. Phys. A: Math. Gen. 34, 5923-5935 (2001). 
[7] J. C. Garrison and J. Wong, "Canonically conjugate pairs, uncertainty relations, and phase operators," J. Math. Phys. 11, 2242-2249 (1970).

[8] A. Galindo, "Phase and number," Lett. Math. Phys. 8, 495-500 (1984).

[9] W. Mlak, "Notes on quantum circular operators," Institute of Mathematics, Polish Academy of Sciences, Preprint 303 (1984).

[10] G. Cassinelli, E. De Vito, P. Lahti, and J.-P. Pellonpää, "Covariant localizations in the torus and the phase observables," quant-ph/0105079, J. Math. Phys., in the press.

Department of Physics, University of Turku, 20014 Turku, Finland

E-mail address: juhpello@utu.fi 